\title{
Echocardiographic Image Sequence Compression Based on Spatial Active Appearance Model
}

\author{
Sándor M. Szilágyi ${ }^{1}$, László Szilágyi ${ }^{1,2}$, and Zoltán Benyó \\ ${ }^{1}$ Sapientia - Hungarian Science University of Transylvania, \\ Faculty of Technical and Human Science, Târgu-Mureş, Romania \\ szs@ms.sapientia.ro \\ 2 Budapest University of Technology and Economics, \\ Dept. of Control Engineering and Information Technology, Budapest, Hungary
}

\begin{abstract}
This paper presents a new method for echocardiographic image sequence compression based on active appearance model. The key element is the intensive usage of all kind of a priori medical information, such as electrocardiography (ECG) records and heart anatomical data that can be processed to estimate the ongoing echocardiographic image sequences. Starting from the accurately estimated images, we could obtain lower amplitude residual signal and accordingly higher compression rate using a fixed image distortion. The realized spatial active appearance model provides a tool to investigate the long term variance of the heart's shape and its volumetric variance over time.
\end{abstract}

Keywords: Echocardiography, active appearance model, image compression, QRS clustering.

\section{Introduction}

Echocardiography is a popular medical imaging modality due to its noninvasive and versatile behavior. There are no known side effects, and the measuring equipment is small and inexpensive relative to other options, such as MRI or CT. Reducing storage requirements and making data access user friendly are two important motivations for applying compression to ultrasound images, with the retention of diagnostic information being critical [2]. A typical echocardiography image consists of a non-rectangular scanned area, and a passive background, which may contain patient related text or limited graphics (e.g. a single channel ECG signal). The resulting spatial variation in image statistics presents a hard task to coding methods that use a single partition strategy. For example, many modern image compression algorithms, such as zero-tree coding [16] and set partitioning in hierarchical trees (SPIHT) 815] are based on the wavelet transform, which partitions the input images into frequency bands whose size decreases logarithmically from high frequencies to low ones. This kind of decomposition strategy works well when the input images are statistically homogeneous, but not in the case of echocardiography image sequences.

In paper [5] is presented the investigation results of the improved transformation based lossy compression techniques for ultrasound and angiography images. 
The goal of the study was to clarify, where the compression process could be improved for the medical application. It was proved that wavelet transform outperforms discrete cosine transform applied to ultrasound image sequence. The analysis of international image compression standards was carried out with special attention to the new still image compression standard: Joint Photographic Experts Group (JPEG) 2000. The JPEG2000 compression is better than ordinary JPEG at higher compression ratio levels. However, some findings revealed this consideration is not necessarily valid for lower compression levels. In study [17. the qualities of the compressed medical images such as computed radiography, computed tomography head and body, mammographic, and magnetic resonance T1 and T2 images were estimated using both methods. The negative effect of blocking artifacts from JPEG was decreased using jump windows.

The impact of image information on compressibility and degradation in medical image compression was presented in paper [7. This study used axial computed tomography images of a head. Both JPEG and JPEG 2000 compression methods were evaluated using various compression ratios (CR) and minimal image quality factor (MIQF).

In pattern recognition research the dimensionality reduction techniques are widely used since it may be difficult to recognize multidimensional data when the number of samples in a data set is comparable with the dimensionality of data space. Locality pursuit embedding (LPE) is a recently proposed method for unsupervised linear dimensionality reduction. LPE seeks to preserve the local structure, which is usually more significant than the global structure preserved by principal component analysis (PCA) and linear discriminant analysis (LDA). In paper 25] the supervised locality pursuit embedding (SLPE) is investigated. These dimensionality reduction methods can improve the understanding level of the medical information borrowed in the recorded image sequences [24].

Usually the medical applications do not tolerate much loss in fidelity, so the distortion free methods, such as context-based adaptive lossless image coding (CALIC) 22] have been recently adapted to "near-lossless" situations 23] with good results. Erickson et al. 4] have compared SPIHT and JPEG methods to compress magnetic resonance imaging (MRI) and ultrasound images. They concluded that wavelet-based methods are subjectively far superior to JPEG compressed at moderately high bit rates. Medical images are typically stored in databases, so it is possible for computers to extract patterns or semantic connections based on a large collection of annotated or classified images. Such automatically extracted patterns can improve the processing and classifying performance of the computers.

In the recent past, researchers in the image analysis community have successfully used statistical modeling techniques to segment, classify, annotate and compress images. Particularly, variations of hidden Markov models (HMMs) have been developed and successfully applied for image and video processing. The key issue in using such complex models is the estimation of system parameters, which is usually a computationally expensive task. In practice, often a tradeoff is accepted between estimation accuracy and running time of the parameter 
estimation method 9]. The matching pursuit (MP) signal coding technique was developed by Mallat and Zhang [12] and enhanced to code the motion prediction signal by Neff and Zakhor [14. The task of the MP coder is to measure the energy of different subunits, each of which represents a motion residual.

Such a statistical information-based estimation highly depends on biological parameters. In our case, the most important task in efficient echocardiography image compression is the accurate detection of QRS complexes from the simultaneously measured ECG signal. Due to the semi-periodic behavior of the ECG signal and echocardiography image sequences, the parameters of the patient model can be more precisely estimated.

Active appearance models (AAM), introduced by Cootes et al. [3], are promising image segmentation tools that may provide solutions to most pending problems of echocardiography, as they rely on both shape and appearance (intensity and/or texture) information. Bosch et al. [1] proposed a robust and timecontinuous delineation of 2-D endocardial contours along a full cardiac cycle, using an extended AAM, trained on phase-normalized four-chamber sequences.

An accurate investigation of the physiology and patho-physiology of the heart, besides studying the electrical activity and spatial distribution of its structures, must also consider the movement of these structures during normal and abnormal cardiac cycles. In this order, simultaneous ECG signal and ultrasound image sequence recording is proposed [20, which reliably supports the localization of the investigated events.

Several papers have already reported the usage of spatial AAM [1318]. The present work has the following contributions: (1) we developed a heart reconstruction algorithm including time-dependent wall boundaries, to estimate the image variances, that allow a better compression rate than conventional methods at a fixed image quality; (2) reported techniques classify ultrasound images only as belonging to systolic or diastolic interval. Our approach distinguishes normal and extra beats, and processes the corresponding images accordingly.

\section{Materials and Methods}

Simultaneous echocardiography sequence recording and ECG signal measurement were carried out at the County Medical Clinic of Târgu-Mureş, using a 2-D echocardiograph that produces 30 frames per second, and a 12-lead ECG monitoring system that samples at $500 \mathrm{~Hz}$ frequency and 12-bit resolution. Each image frame received a time stamp, which served for synchronization with ECG events. Two different series of measurements were recorded. The first series, which served for AAM training, consisted of 35 patients (12 of whom having extraventricular beats), 20 ultrasound sequences for each patient, of 10-15 seconds length each, with previously established transducer placements. Based on these data, an a priori information database was created, which organized the ultrasound images grouped by corresponding ECG events.

The second series of measurements, which involved 8 patients, consisted of two stages. In the first stage, the same measurements were performed, as in the 
first series, in order to provide patient-specific training data for the AAM. In the second stage, several measurements of the same individuals but recorded in different moments were performed using different placements and positions of the transducer. In this order, image sequences were recorded at 8 parallel cross sections in horizontal and rotated $\left(45^{\circ}\right.$ to the left and to the right) positions with a $1 \mathrm{~cm}$ inter-slice distance. We used 10 common axis planes that were placed at the front, lateral and back side of the torso, resulting for each patient $10 \times 8 \times 3=240$ image sequences of $2-3$ seconds duration.

The duration of the recorded image sequences was restricted by the semiperiodic behavior of the ECG signal. The spatial movement of the heart is constrained by the course of the depolarization-repolarization cycle [19]. The studied ECG parameters were: shape of QRS beat, QT and RR distances. These parameters characterize the nature of a QRS complex, and were determined as presented in [19]. ECG event clustering was accomplished using Hermite functions and self-organizing maps [10. Two main event clusters were created: normal and ventricular extra beats. This latter group, because of the patient specific manifestation of ventricular extras, had to be dealt with separately patient by patient. QRS beats not belonging to any cluster were excluded from further processing, together with their corresponding ultrasound sub-sequences. A further condition for normal QRS complexes to be included was having RR distance between 700-800 ms and QT distance between 350-400 ms. A detailed presentation of ECG processing is presented in Fig. 1.

The time-varying evolution of the cardiac volume is determined by the interconnection of electrical and mechanical phenomena. In a whole cardiac cycle there are two extremity values. The maximal volume can be coupled with the starting moment of ventricular contraction. The moment of minimal volume shortly precedes the termination of ventricular contraction, but is much more difficult to identify, due to the dead time of a normal cardiac cell. This delay is caused by the behavior of a regular cardiac cell, whose electric response precedes with 60-80 ms the mechanical contraction [21. The combination of the electrical and mechanical properties of the heart and the usage of knowledge-base allowed us to create a performance evaluation module, shown in Fig. 2 that determines the most probable wall position. This image presents an overview of the image processing and volumetric reconstruction procedure. The first algorithmic step is noise elimination. Speckle noise represents a major difficulty to most ultrasound imaging applications [6]. In our case, the suppression of such phenomena was accomplished using the well-known motion adaptive spatial technique presented in 20]. Due to the measuring technique of traditional echocardiography, the obtained images are distorted. In order to become suitable input for 3-D processing, they need to go through a normalization transform. Every recorded ultrasound slice is represented by a plane, whose spatial alignment depends on the position and rotational angle of the transducer. The normalization process also takes into consideration the distance of each image pixel from the transducer.

The AAM was trained using the spatial position of each slice recorded in the first measurement series. By averaging these spatial distributions, a mean 4-D 


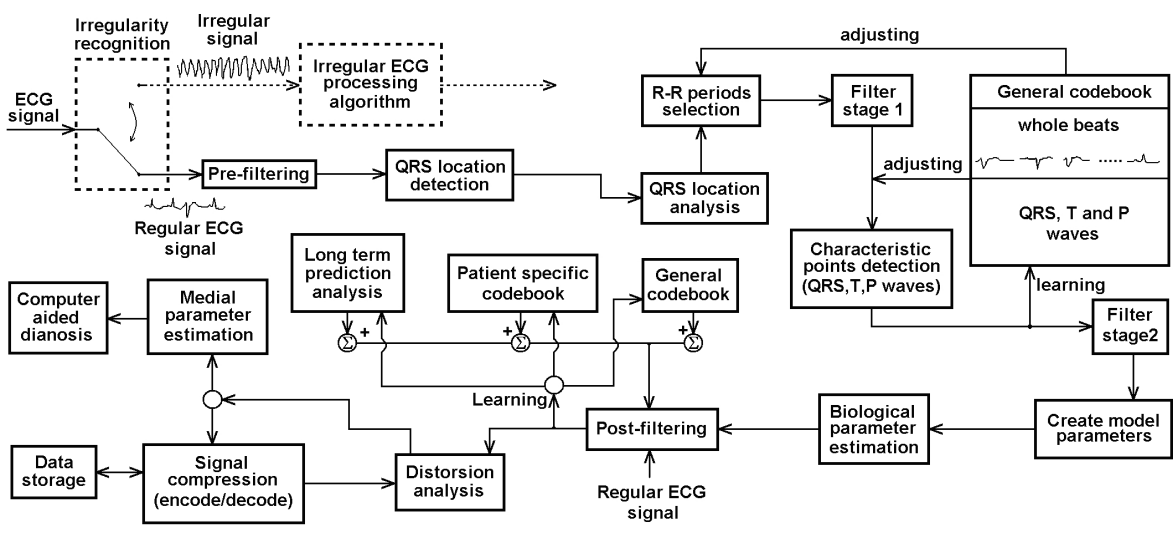

Fig. 1. The proposed ECG signal filtering, processing and compression

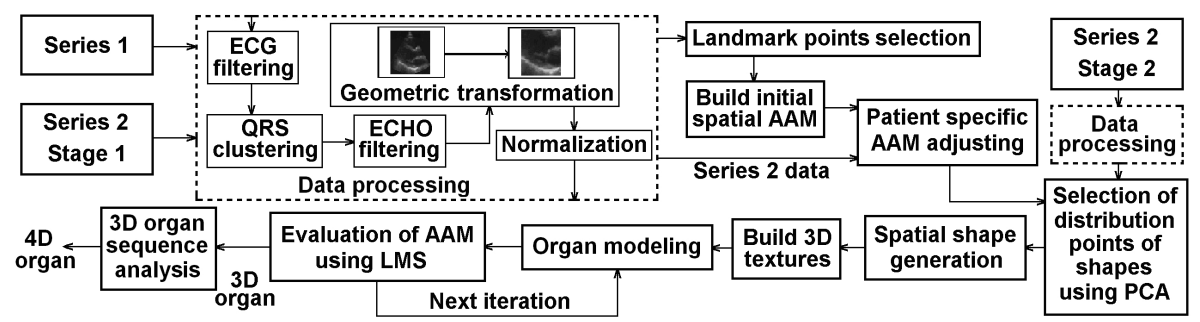

Fig. 2. The data recording and analyzing procedure: all echocardiography and ECG data go through the same processing module. The AAM is constructed from the measurements of series 1 , and fine tuned afterward using the patient specific data resulting from series 2 stage 1 . Stage 2 data serve for the detailed cardiac volumetric analysis. Reconstructed 3-D objects are finally aligned using an iterative LMS-based algorithm.

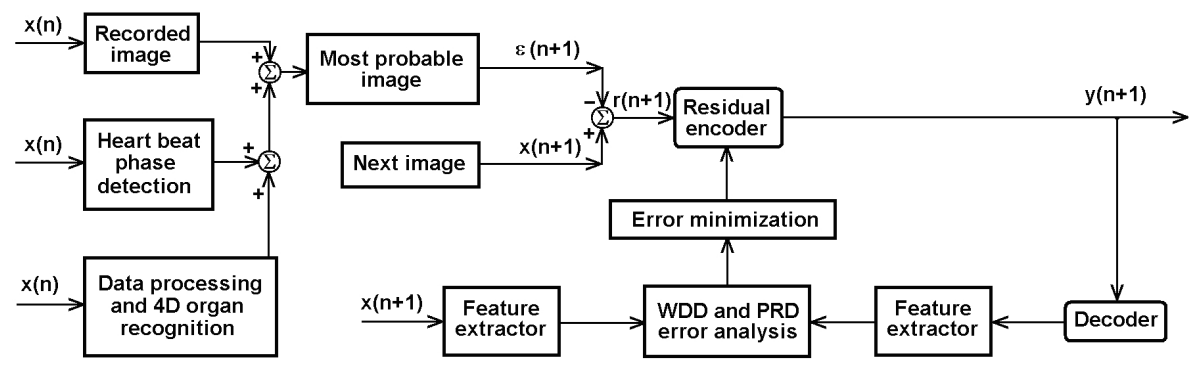

Fig. 3. Residual data construction and distortion analysis using the recorded image, heart beat phase information and AAM-based 4D organ shape to determine the most probable image in the next cycle. As the $y(n+1)$ output signal is obtained, a WDD and PRD-based distortion analysis is performed, using image features given by AAM. 
heart shape model is obtained, which will be the base shape of the AAM [111]. Landmark points are determined as by Mitchell et al. [13. The sparse character of the obtained spatial description model doesn't allow the landmark points have 3-D texture information, so we restricted the texture to 2-D.

Subjects have their own specific, time-dependent inner structure, which cannot be properly approximated from a population of few dozens of individuals. In order to make further adjustments to the AAM, the base structure was adjusted to the patient using the measurements made in the second series, first stage. The landmark points determined on the images recorded during the second stage of the second series allowed us to create a $3-\mathrm{D}$ distribution point model, which was established according to 13 . Having the distribution points established, the AAM will be enabled to adjust itself to a diversity of biological factors like the phase of ECG and breathing. A detailed description of the manifestation of these phenomena and the model adaptation is given in [18]. Our algorithm acts similarly, but it treats the cardiac cycle differently: not only systolic and diastolic phases are distinguished, but also a QRS complex clustering is performed to give different treatment to normal and ventricular cardiac cycles.

Spatial texture maps are determined via averaging [18. The visual aspect of the heart and its environment, because of their mutual motion, is changing in time. AAM models only include information on the texture situated within the model. The time dependent representation of the ultrasound slices obtained from the large stack of sequences enabled us to accurately determine the 4-D structure of the heart [13. The iterative algorithm of the AAM demands the comparison of measured and expected shapes. The AAM was adjusted using a quadratic cost function, until the desired accuracy was obtained.

Due to its adaptive behavior, the compression method can handle patientdependent data and efficiently separate the measured artifacts from the useful signal. The proposed signal compression algorithm consists of the following steps:

- Intelligent image sequence analysis and filtering, that involves the automatic recognition of echocardiography image, ECG signal and various changing and constant labels and letters that appear on the recorded image sequence;

- Background selection (the constantly dark region);

- ECG signal processing (see Fig. 1);

- Segmentation of ultrasound image (see Fig. 2);

- Calculation of the heart's 4-D shape (3-D + time) using AAM;

- Estimation of probable image;

- Residual signal estimation, 2-way entropy coding and back-estimation.

Compression results were evaluated using the percentage root mean square difference (PRD) and weighted diagnostic distortion (WDD) [26]. WDD measures the relative preservation of the diagnostic information (e.g. location, duration, intensity, shapes, edges) in the reconstructed image. These diagnostic features were determined by physicians. The distortion estimation and signal (ultrasound image and all auxiliary data) coding process is presented in Fig. 3 . 


\section{Results}

Figure4(a) presents two series of ultrasound slices indicating the contour of the left ventricle of the 2nd patient, detected during a ventricular contraction. The two columns of slices show two different views, having $60^{\circ}$ angle difference. The four slices in each column represent subsequent images of the sequence, showing the approximately $100 \mathrm{~ms}$ duration of a ventricular contraction. Figure 4 (b) exhibits the same ultrasound slices after being normalized, that is, transformed to Euclidean space. Figure 4(c) shows two different reconstructed 3-D shapes of the left ventricle.

Figure 5 presents the decoded echocardiography images at various compression rates. The variation of RMSE level against inverted compression rate is shown in Fig. 5(e). The RMSE graph was created for an averaged normal QRS beat shape, and average RR and QT distances.
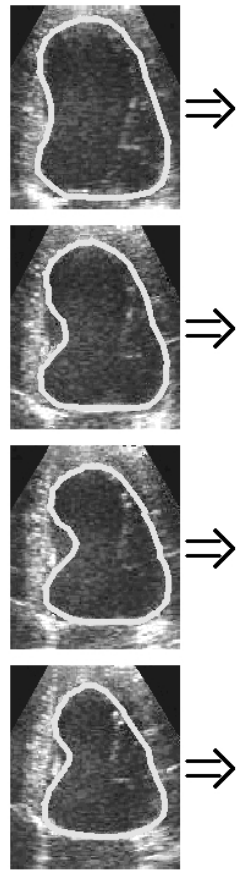

(a)
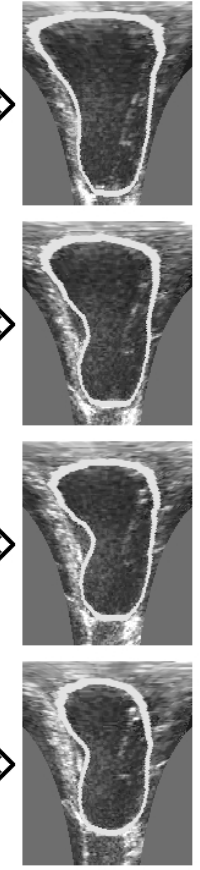

(b)
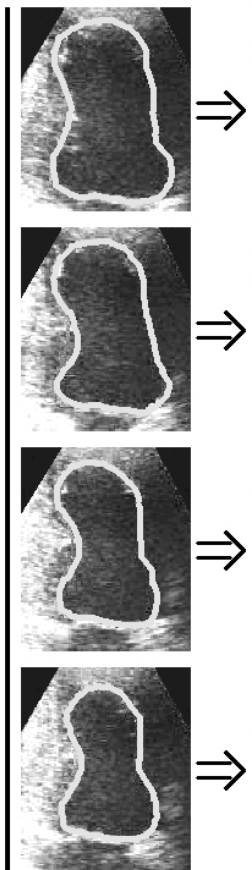

(a)
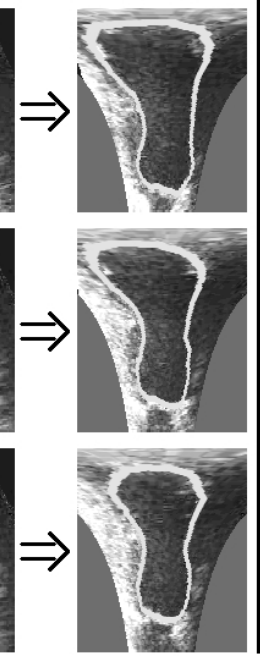

(b)
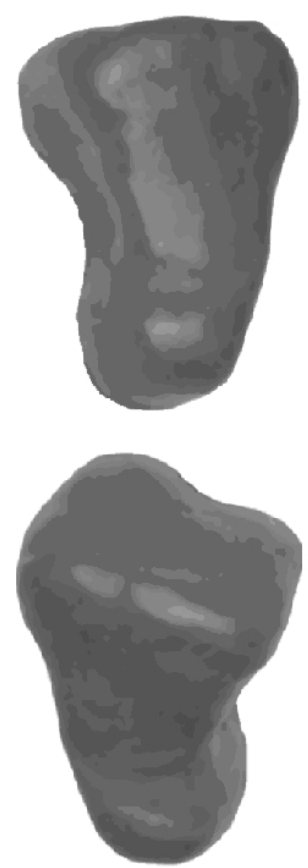

(c)

Fig. 4. Results of shape reconstruction: (a) and (b) time varying 2-D contour of the left ventricle before and after normalization, (c) reconstructed 3-D structure of the left ventricle

\section{Discussion}

The recognition of the relation between echocardiography images and simultaneously recorded ECG signal is a key element in efficient image sequence 


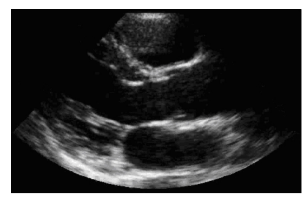

(a)

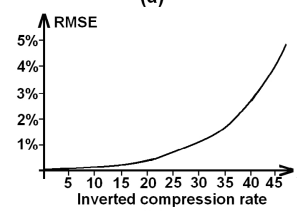

(e)

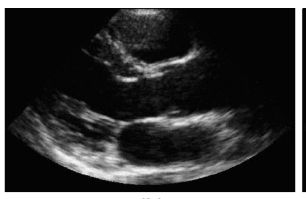

(b)

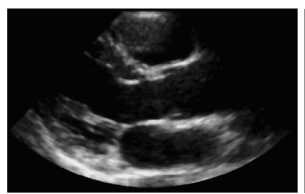

(f)

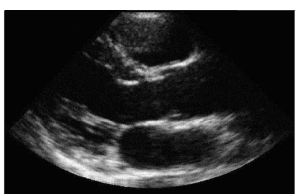

(c)

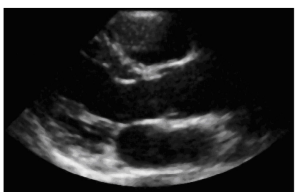

(g)

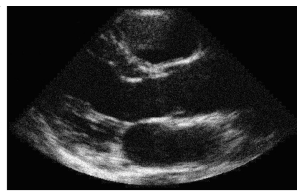

(d)

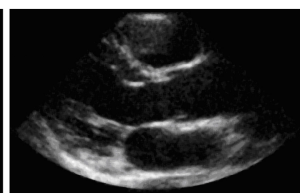

(h)

Fig. 5. Results of image reconstruction: (a) original image, (b) decoded image at 1/22 compression rate, (c) decoded image at 1/39 compression rate, (d) decoded image at 1/54 compression rate, (e) representation of root mean square error (RMSE) vs. inverted compression rate, (f) feature-base corrected image at $1 / 22$ compression rate, (g) feature-base corrected image at 1/39 compression rate, (h) feature-base corrected image at $1 / 54$ compression rate

compression (see Table1). However, various events like aspiration and expiration may influence the measured data. During a whole cardiac cycle, the shape and volume of the left ventricle changes considerably. It is difficult to determine the performance of the reconstruction method for the sporadically occurred ventricular extra-systolic beats. Even for patients that produce at least five extra beats with similar shapes in each minute the reconstruction performance remains well below the normal QRS cluster's accuracy, due to the sparse distribution of the processable slices.

From Fig. 5 we can observe that even a well-working WDD correction method cannot handle a compression rate better than 1/60 without a serious image distortion that can lead to wrong medical diagnosis. Table 1 demonstrates the higher performance of the proposed image compression method, that exists due

Table 1. The obtained inverted compression rates obtained for normal beats, at various RMSE levels, using the following methods: Wavelet-based compression, Image comparison-based estimation, ECG and echocardiography image compression using QRS long-term prediction (LTP), JPEG 2000, Matching Pursuits, proposed AAMbased compression

\begin{tabular}{llllll}
\hline Compression Method vs. RMSE & $1 \%$ & $2 \%$ & $3 \%$ & $4 \%$ & $5 \%$ \\
\hline Wavelet-based compression [8] & 10.2 & 15.4 & 17.3 & 18.8 & 19.9 \\
Image comparison [22] & 14.2 & 21.2 & 24.0 & 26.1 & 27.8 \\
QRS and LTP image comparison [26] & 17.4 & 26.6 & 29.6 & 31.8 & 33.7 \\
JPEG 2000 & 13.7 & 20.5 & 23.2 & 25.1 & 26.6 \\
Matching pursuits [12] & 15.7 & 22.9 & 27.1 & 29.5 & 31.4 \\
Proposed AAM-based & 22.7 & 36.3 & 40.2 & 44.1 & 47.7 \\
\hline
\end{tabular}


to the advanced QRS beat analysis and spatial AAM-based organ reconstruction. Such an analysis can lead to a much better 'estimated image' quality that reduces the amplitude of the residual signal. The improvement brought by the proposed method is more relevant in case of normal beats, because the AAM can better adapt itself to their characterization due to their higher incidence.

\section{Conclusions}

The investigation of simultaneously recorded ECG and echocardiography images enables us to study the relations between the electrical and mechanical phenomena concerning the heart. The signal and image estimation is made by using various priori medical information, and the algorithm yields lower amplitude residual signal and better compression ratio at given distortion level.

The compression method presented in this paper performs well in case of normal and quite well for ventricular beats. The presence of pathological image and signal samples may lower the performance difference among the proposed method and the tested algorithms. This kind of approach of the problem may result in deeper understanding of electrical and mechanical properties of the heart, that provides a much efficient compression than other algorithms using less a priori information.

Acknowledgements. This research was supported by the Sapientia Institute for Research Programmes.

\section{References}

1. Bosch, J.G., Mitchell, S.C., Lelieveldt, B.P.F., Nijland, F., Kamp, O., Sonka, M., Reiber, J.H.C.: Automatic segmentation of echocardiographic sequences by active appearance motion models. IEEE Trans. Med. Imag. 21, 1374-1383 (2002)

2. Chiu, E., Vaisey, J., Atkins, M.S.: Wavelet-Based Space-Frequency Compression of Ultrasound Images. IEEE Trans. Inf. Tech. Biomed. Eng. 5, 300-310 (2001)

3. Cootes, T.F., Edwards, G.J., Taylor, C.J.: Active appearance models. IEEE Trans. Patt. Anal. Mach. Intell. 23, 681-685 (2001)

4. Erickson, B.J., Manduca, A., Palisson, P., Persons, K.R., Earnest, D., Savcenko, V.: Wavelet compression of medical images. Radiology 206, 599-607 (1998)

5. Erickson, B.J.: Irreversible Compression of Medical Images. J. Digit. Imag. 15, 5-14 (2002)

6. Evans, A.N., Nixon, M.S.: Biased motion-adaptive temporal filtering for speckle reduction in echocardiography. IEEE Trans. Med. Imag. 15, 39-50 (1996)

7. Fidler, A., Skaleric, U.: The impact of image information on compressibility and degradation in medical image compression. Med. Phys. 33, 2832-2838 (2006)

8. Hang, X., Greenberg, N.L., Zheng, Y.F., Thomas, J.D.: Compression of 3-D echocardiographic images using a modified 3-D set-partitioning-in-hierarchicaltrees algorithm based on a 3-D wavelet packet transform. J. Electr. Imag. 15, 1-13 (2006) art. no. 023016

9. Joshi, D., Li, J., Wang, J.Z: A Computationally Efficient Approach to the Estimation of Two- and Three-Dimensional Hidden Markov Models. IEEE Trans. Imag. Proc. 15, 1871-1886 (2006) 
10. Lagerholm, M., Peterson, C., Braccini, G., Edenbrandt, L., Sörnmo, L.: Clustering ECG complexes using Hermite functions and self-organizing maps. IEEE Trans. Biomed. Eng. 47, 838-848 (2000)

11. Lelieveldt, B.P.F., van der Geest, R.J., Mitchell, S.C., Bosch, J.G., Sonka, M., Reiber, J.H.C.: 3-D active appearance models: fully automatic detection of endoand epicardial contours in short-axis cardiac MR data. Proc. Int. Soc. Magn. Res. Med (ISMRM) 2, 1668 (2002)

12. Mallat, S., Zhang, Z.: Matching pursuits with time-frequency dictionaries. IEEE Trans. Signal Proc. 41, 3397-3415 (1993)

13. Mitchell, S.C., Bosch, J.G., Lelieveldt, B.P.F., van der Geest, R.J., Reiber, J.H.C., Sonka, M.: 3-D active appearance models: segmentation of cardiac MR and ultrasound images. IEEE Trans. Med. Imag. 21, 1167-1178 (2002)

14. Neff, R., Zakhor, A.: Very low bit rate video coding based on matching pursuits. IEEE Trans. Circ. Syst. Video Techn. 7, 158-171 (1997)

15. Said, A., Pearlman, W.A.: A new, fast, and efficient image codec based on set partitioning in hierarchical trees. IEEE Trans. Circ. Syst. Video Techn. 6, 243-250 (1996)

16. Shapiro, J.: Embedded image coding using zerotrees of wavelet coefficients. IEEE Trans. Sign. Proc. 41, 3445-3462 (1993)

17. Shiao, Y.H., Chen, T.J., Chuang, K.S., Lin, C.H., Chuang, C.C.: Quality of Compressed Medical Images. J. Digit. Imag. 20, 149-159 (2007)

18. Stegmann, M., Pedersen, D.: Bi-temporal 3D active appearance models with applications to unsupervised ejection fraction estimation. Progr. Biomed. Opt. Imag. Proc. SPIE 5746, 336-350 (2005)

19. Szilágyi, S.M., Szilágyi, L., Benyó, Z.: Support Vector Machine-Based ECG Compression. Ser. Adv. Soft Comput (IFSA 2007) 41, 737-745 (2007)

20. Szilágyi, S.M., Szilágyi, L., Benyó, Z.: Volumetric Analysis of the Heart Using Echocardiography. In: FIMH 2007. LNCS, vol. 4466, pp. 81-90 (2007)

21. Winslow, R.L., Hinch, R., Greenstein, J.L.: Mechanisms and models of cardiac excitation-contraction coupling. Lect. Notes Math. vol. 1867, pp. 97-131 (2005)

22. Wu, X., Memon, N.: Context-based adaptive lossless image coding. IEEE Trans. Comm. 45, 437-444 (1997)

23. Wu, X., Bao, P.: L-infinity constrained high-fidelity image compression via adaptive context modeling. IEEE Trans. Imag. Proc. 9, 536-542 (2000)

24. Yuan, Y., Evans, A.N., Monro, D.M.: Low Complexity Separable Matching Pursuits. In: Proc. IEEE Int. Conf. Acoust. Speech Sign. Proc. III, pp. 725-728. IEEE Computer Society Press, Los Alamitos (2004)

25. Zhenga, Z., Yang, J.: Supervised locality pursuit embedding for pattern classification. Imag. Vis. Comp. 24, 819-826 (2006)

26. Zigel, Y., Cohen, A., Katz, A.: ECG Signal Compression Using Analysis by Synthesis Coding. IEEE Trans. Biomed. Eng. 47, 1308-1316 (2000) 